\title{
Perfil Epidemiológico de traumas ortopédicos pediátricos em um hospital do interior do Pará
}

\author{
Epidemiological profile of pediatric orthopedic trauma in a hospital in the interior of Pará \\ Perfil epidemiológico del traumatismo ortopédico pediátrico en un hospital del interior de \\ Pará
}

Benedito do Carmo Gomes Cantão ${ }^{1 *}$, Ailson Almeida Veloso Júnior ${ }^{1}$, Eunice Lara dos Santos Cunha ${ }^{1}$, José Benedito dos Santos Batista Neto ${ }^{2}$, Cristielle Larissa Sousa de Almeida ${ }^{2}$, Herberth Rick dos Santos Silva ${ }^{2}$, Caio Heitor Vieira Melo², Alan Barros de Alencar ${ }^{3}$, Genislaine Ferreira Pereira ${ }^{4}$, Anderson Bentes de Lima ${ }^{5}$.

\section{RESUMO}

Objetivo: Descrever o perfil epidemiológico dos pacientes de traumas ortopédicos pediátricos, que passaram por procedimento cirúrgico em um hospital de alta-média complexidade. Métodos: Trata-se de um estudo quantitativo epidemiológico, de caráter descritivo e corte transversal retrospectivo. A pesquisa contou com o registro de 643 pacientes ortopédicos pediátricos atendidos no hospital entre janeiro de 2017 a dezembro de 2018. Resultados: Acerca do sexo biológico, a maior parte dos pacientes constituiu-se de meninos (58,32\%), enquanto meninas corresponderam a $41,68 \%$ dos casos. Quando separados por idade, o intervalo dominante foi composto por crianças de $5-12$ anos $(79,77 \%)$. Os membros superiores foram os mais afetados, com maior prevalência de fraturas de rádio, $216(33,49 \%)$, seguido pelo acometimento do antebraço, associação entre os ossos rádio e ulna, do qual foram registrados $154(23,88 \%)$ casos e 66 $(10,24 \%)$ fraturas de úmero. Por conseguinte, acerca dos membros inferiores, os ossos mais afetados foram a tíbia com 51 (7,9\%) casos e o fêmur com 35 (5,43\%). Conclusão: O objetivo do estudo foi alcançado, sendo possível traçar o perfil epidemiológico dos pacientes pediátricos acometidos por traumas ortopédicos e atendidos na instituição, durante os anos de 2017 e 2018.

Palavras-chave: Ortopedia, Epidemiologia, Lesões.

\begin{abstract}
Objective: To describe the epidemiological profile of pediatric orthopedic trauma patients who underwent a surgical procedure in a high-medium complexity hospital. Methods: This is a quantitative, epidemiological, descriptive and retrospective cross-sectional study. The survey counted on the registration of 643 pediatric orthopedic patients treated at the hospital between January 2017 and December 2018. Results: Regarding biological sex, most patients were boys (58.32\%), while girls accounted for $41.68 \%$ of cases. When separated by age, the dominant range was composed of children aged $5-12$ years $(79.77 \%)$. The upper limbs were the most affected, with a higher prevalence of radius fractures, 216 (33.49\%), followed by the involvement of the forearm, an association between the radius and ulna bones, of which $154(23.88 \%)$ cases were recorded and $66(10.24 \%)$ humerus fractures. Therefore, concerning the lower limbs, the bones most affected were the tibia with $51(7.9 \%)$ cases and the femur with $35(5.43 \%)$. Conclusion: The objective of the study was achieved, making it possible to trace the epidemiological profile of pediatric patients affected by orthopedic trauma and treated at the hospital, during the years 2017 and 2018.
\end{abstract}

Keywords: Orthopedics, Epidemiology, Injuries.

\footnotetext{
${ }^{1}$ Universidade do Estado do Pará (UEPA), Belém - PA. * E-mail: bcgcantao@hotmail.com

2 Universidade do Estado do Pará (UEPA), Tucuruí - PA.

${ }^{3}$ Santa Casa de Misericórdia de Franca, Franca - SP.

${ }^{4}$ Secretaria Municipal de Saúde (SMS), Tucuruí - PA.

5 Universidade Federal do Pará (UFPA), Belém - PA.
} 


\section{RESUMEN}

Objetivo: Describir el perfil epidemiológico de los pacientes pediátricos con traumatismo ortopédico sometidos a un procedimiento quirúrgico en un hospital de complejidad media-alta. Métodos: Se trata de un estudio transversal cuantitativo, epidemiológico, descriptivo y retrospectivo. La encuesta contó con el registro de 643 pacientes ortopédicos pediátricos atendidos en el hospital entre enero de 2017 y diciembre de 2018. Resultados: En cuanto al sexo biológico, la mayoría de los pacientes fueron niños (58,32\%), mientras que las niñas representaron el $41,68 \%$ de los casos. Cuando se separan por edad, el rango dominante lo componen los niños de 5 a 12 años (79,77\%). Los miembros superiores fueron los más afectados, con una mayor prevalencia de fracturas de radio, $216(33,49 \%)$, seguido de la afectación del antebrazo, asociación entre el radio y el cúbito, de los cuales se registraron $154(23,88 \%)$ casos. y 66 $(10,24 \%)$ fracturas de húmero. Por tanto, en los miembros inferiores, los huesos más afectados fueron la tibia con $51(7,9 \%)$ casos y el fémur con 35 (5,43\%). Conclusión: Se logró el objetivo del estudio, posibilitando trazar el perfil epidemiológico de los pacientes pediátricos afectados por traumatismo ortopédico y atendidos en el hospital, durante los años 2017 y 2018.

Palabras clave: Ortopedia, Epidemiología, Lesiones.

\section{INTRODUÇÃO}

A ortopedia é uma especialidade da área das ciências da saúde relacionada ao processo de prevenção, tratamento e reabilitação de enfermidades relacionadas ao aparelho locomotor. Associado a esta especialidade, temos também a traumatologia, a qual lida, especificamente, com pacientes vítimas de traumas, no geral (SILBERMAN FS e VARAONA O, 2018).

Acerca do trauma, temos que este é definido como um evento nocivo constituído por alterações estruturais e físicas, ou um desequilíbrio homeostático advindo de uma troca entre o tecido e o meio. É tido como uma patologia que compromete o bem-estar do indivíduo, interferindo na esfera econômica e social, deste, na sociedade. Para mais, tem-se que, por ano, cerca de 60 milhões de indivíduos são acometidos por alguma lesão traumática, o que nos permite inferir que para cada seis hospitalizações, uma está relacionada ao referido impasse (SANTOS LFS, et al., 2016). Assim sendo, nos dias de hoje, esta problemática é uma das principais causas de morte nos primeiros 40 anos de vida (SILVA LAP, et al., 2017).

Ademais, de acordo com Silva LAP, et al. (2017), o desenvolvimento industrial e tecnológico, possibilitou um aumento considerável de frotas veiculares, assim como o aumento da civilização, levando a um complexo processo de alta da violência urbana. Por conseguinte, os processos, antes citados, coincidiram no aumento exponencial de um grave problema de saúde: os traumas ortopédicos, dentre eles, o infantil.

Segundo Castro RRM, et al. (2013), os eventos de cunho traumático, envolvendo fraturas e outras patologias ortopédicas, já são o principal agravo de saúde que acomete a população de $0-39$ anos no Brasil, tornando-se um problema de saúde pública, principalmente, ao consideramos as implicações físicas e psicológicas impostas as crianças e jovens, faixa-etária, esta, potencialmente produtiva.

Em seu estudo, Souza DFM, et al. (2010), alega que nos Estados Unidos da América, uma a cada três crianças é, por ano, atingida por alguma espécie de traumatismo ortopédico. Sendo assim, o traumatismo é responsável por entre $10 \%$ a $15 \%$ das internações em clínicas pediátricas hospitalares e em unidades de terapia intensiva. $\mathrm{O}$ estudo dos autores antes citados ainda alega que para cada indivíduo infantil que vêm a óbito, 40 crianças requerem hospitalização, 1000 requerem tratamento de emergência e algumas dezenas sofrem de incapacidades permanentes.

Por consequência, dentre os prognósticos dos traumas ortopédicos, estão as amputações. Chamlian TR, et al. (2013), apontam que as amputações, no geral, não afetam somente o corpo físico do paciente, mas também sua integralidade psicológica, social e individual. Além disso, a pesquisa dos autores ainda mostra que somente nos Estados Unidos da América, são demonstrados 50 mil novos casos de amputação, envolvendo fatores traumáticos, por ano, e no Brasil, cerca de 13.9 a cada 100 mil habitantes, somente no estado do Rio de Janeiro. 
Para mais, o estudo de Almeida M (2019), relata que na Europa a principal causa de morte ou incapacitação de crianças e jovens de 0 a 18 anos é o traumatismo. $O$ autor afirma que os acidentes pediátricos ocorrem principalmente em escolas, seguido da casa do indivíduo e de lugares públicos. A importância de conhecer a epidemiologia do trauma infantil, dá-se no objetivo de fornecer prevenção fundamentada, neste âmbito.

Crianças e adolescentes, são mais propensos aos traumas ortopédicos, tendo em vista sua menor estatura quando em comparação a de um adulto, de tal maneira, sendo expostos, por vezes, à múltiplas lesões. Portanto, a coleta de dados específicos, no que tange aos fatores envolvidos no trauma, bem como, as partes anatômicas afetadas, são de suma importância na literatura, para a obtenção de melhores protocolos de saúde e prevenção (ARAÚJO NR, 2014).

Ademais, crianças estão mais predispostas à desenvoltura e acometimento de fraturas ortopédicas, também, por seu menor discernimento perante os perigos e agentes etiológicos envolvidos no traumatismo, aliado ao constante comportamento inquieto, exploratório e aventureiro, comum para a idade, onde descobre-se pessoalmente os perigos do mundo ao seu redor. Ainda, os mesmos tendem a ter maior nível de energia para ser gasta pelo organismo, resultando em comportamentos perigosos para sua saúde (PEREIRA IF, et al., 2013).

Nesse cenário, o desenvolvimento de pesquisas clínicas e/ou epidemiológicas dos pacientes acometidos por traumas ortopédicos, em especial o público infantil, é fundamental para a saúde pública de nosso país, tendo em vista que servirão como justificativa para o planejamento, organização e realização de estratégias por parte dos governos federal, estaduais e/ou municipais que visem a diminuição desses agravos, uma vez que será conhecida uma determinada realidade local. Tal diminuição, acarretará na redução da demanda em hospitais, no número de vítimas precoces, bem como diminuirá os gastos com assistência, que em questões ortopédicas é uma das maiores (SOUSA LRB, et al., 2017).

A partir do que fora citado anteriormente, o presente estudo tem como objetivo descrever o perfil epidemiológico dos pacientes de traumas ortopédicos pediátricos, que passaram por procedimento cirúrgico em um hospital de alta-média complexidade.

\section{MÉTODOS}

Trata-se de um estudo quantitativo epidemiológico, de caráter descritivo e corte transversal retrospectivo, desenvolvido em um Centro Cirúrgico de um hospital do interior do estado do Pará no ano de 2020. O referido hospital é uma instituição de média e alta complexidade, sendo referência no serviço de urgência e emergência, atendendo, portanto, diversas regiões do estado e, em algumas ocasiões, de fora do estado.

A população estudada compreende os pacientes ortopédicos pediátricos, de 0 a 12 anos, registrados no hospital e que passaram por procedimentos cirúrgicos ortopédicos com diagnóstico de lesão traumática comprovada. Esses indivíduos possuem seus dados registrados no "Livro de Registros de Cirurgias Ortopédicas" do Centro Cirúrgico, sendo este a fonte de extração de dados da pesquisa.

Por conseguinte, no livro são registradas as seguintes informações de pacientes: sexo biológico, idade, procedência e partes do corpo afetadas, sendo estas, portanto, as variáveis do presente estudo. Acerca da coleta de dados, esta foi realizada no ano de 2020. Ademais, foram coletados os dados dos pacientes atendidos dentro do período de janeiro de 2017 a dezembro de 2018.

Após a coleta, os dados foram digitados e agrupados em áreas afins e codificados em planilhas eletrônicas com auxílio dos softwares de computador Microsoft Office Word 2019 e Microsoft Office Excel 2019. A partir disso, foram construídas tabelas com os dados oriundos de operações estatísticas simples (porcentagens), facilitando, assim, a compreensão das informações geradas. Posteriormente, tais resultados foram comparados com outros estudos presentes na literatura.

Por fim, cumprindo a resolução no 466/2012, do Conselho Nacional de Saúde do Ministério da Saúde, a qual trata dos aspectos éticos de pesquisas com seres humanos, a coleta de dados foi realizada somente após aprovação do projeto da pesquisa pelo Comitê de Ética em Pesquisa da Universidade do Estado do Pará (UEPA) pelo parecer no: 4.265.878, CAAE: 32458520.1.000.5170. 


\section{RESULTADOS}

A partir das informações contidas no "Livro de Registros de Cirurgias Ortopédicas" do Centro Cirúrgico, foi verificado que durante o período de janeiro de 2017 a dezembro de 2018, foram atendidos, no Centro Cirúrgico, 1899 pacientes ortopédicos. Destes, 643 foram incluídos no estudo, pois representam os pacientes ortopédicos pediátricos.

Acerca do sexo biológico, a maior parte dos pacientes pediátricos atendidos, no período analisado, constituiu-se de meninos (58,32\%), enquanto meninas correspondeu a $41,68 \%$ dos casos. Quando separados por idade, o intervalo dominante foi composto por crianças de 5 a 12 anos (79,77\%) (Tabela 1).

Tabela 1 - Caracterização dos pacientes pediátricos atendidos no Centro Cirúrgico, no período de 2017 a 2018, quanto às variáveis relativas a sexo e idade.

\begin{tabular}{cll}
\hline Variável & $\mathbf{N}$ & $\%$ \\
\hline Sexo & & \\
\hline Masculino & 375 & 58,32 \\
Feminino & 268 & 41,68 \\
\hline Idade (anos) & & \\
\hline 0 & 11 & 1,72 \\
1 & 20 & 3,11 \\
2 & 22 & 3,43 \\
3 & 39 & 6,07 \\
4 & 38 & 5,90 \\
5 & 62 & 9,64 \\
6 & 69 & 10,73 \\
7 & 70 & 10,88 \\
8 & 53 & 8,24 \\
9 & 62 & 9,64 \\
10 & 67 & 10,42 \\
11 & 70 & 10,88 \\
12 & 60 & 9,34 \\
\hline Total & 673 & $\mathbf{1 0 0} \%$
\end{tabular}

Fonte: Cantão BCG, et al., 2020; dados extraídos do setor de estatística do centro cirúrgico.

Quanto a procedência desses pacientes, a maioria, 249 crianças $(38,73 \%)$, eram do município de Tucuruí, seguido por 127 (19,75\%) de Novo Repartimento, 93 (14,47\%) de Breu Branco, 63 (9,79\%) de Pacajá, $53(8,25 \%)$ de Jacundá, 49 (7,62\%) de Goianésia, $3(0,46 \%)$ de Baião e $1(0,15 \%)$ de Mojú, ainda, existiram $5(0,78 \%)$ pacientes com procedência indeterminada (Tabela 2).

Tabela 2 - Procedência dos pacientes pediátricos atendidos no Centro Cirúrgico.

\begin{tabular}{lll}
\hline Procedência & N & $\%$ \\
\hline Tucuruí & 249 & 38,73 \\
Novo Repartimento & 127 & 19,75 \\
Breu Branco & 93 & 14,47 \\
Pacajá & 63 & 9,79 \\
Jacundá & 53 & 8,25 \\
Goianésia & 49 & 7,62 \\
Baião & 3 & 0,46 \\
Mojú & 1 & 0,15 \\
Indeterminado & 5 & 0,78 \\
\hline Total & $\mathbf{6 7 3}$ & $\mathbf{1 0 0 \%}$
\end{tabular}

Fonte: Cantão BCG, et al., 2020; dados extraídos do setor de estatística do centro cirúrgico. 
No que tange a parte do corpo afetada, houveram 645 fraturas registradas, tendo em vista que a amostra é composta por 643 crianças, foi verificado que 641 tiveram uma única fratura, enquanto 2 tiveram duas fraturas. Ademais, os membros superiores foram os mais afetados, com maior prevalência de fraturas de rádio, $216(33,49 \%)$, seguido pelo acometimento do antebraço, associação entre os ossos rádio e ulna, do qual foram registrados 154 (23,88\%) casos e 66 (10,24\%) fraturas de úmero (Tabela 3).

Por conseguinte, acerca dos membros inferiores, os ossos mais afetados foram a tíbia com 51 (7,9\%) dos casos e o fêmur com 35 (5,43\%). As demais regiões do corpo foram encontradas em menores proporções.

Tabela 3 - Partes do corpo dos pacientes ortopédicos pediátricos atendidos no Centro Cirúrgico, no período de 2017 a 2018, afetadas.

\begin{tabular}{lll}
\hline Parte do corpo afetada & N & $\%$ \\
\hline Rádio & 216 & 33,49 \\
Antebraço & 154 & 23,88 \\
Úmero & 66 & 10,24 \\
Tíbia & 51 & 7,9 \\
Fêmur & 35 & 5,43 \\
Mão & 30 & 4,65 \\
Pé & 28 & 4,35 \\
Tendão Calcâneo & 21 & 3,25 \\
Joelho & 20 & 3,1 \\
Quadril & 16 & 2,48 \\
Fíbula & 7 & 1,08 \\
Clavícula & 1 & 0,15 \\
\hline Total & $\mathbf{6 7 5}$ & $\mathbf{1 0 0 \%}$ \\
\hline
\end{tabular}

Fonte: Cantão BCG, et al., 2020; dados extraídos do setor de estatística do centro cirúrgico.

\section{DISCUSSÃO}

O trauma ortopédico pode ser entendido como sendo um abalo físico proveniente de grandes impactos abruptos ou violência, o que pode ocasionar alterações estruturais, fisiológicas ou psicológicas do organismo afetado (SILVA LA, et al., 2017). Por conseguinte, os traumas ortopédicos são considerados problemas de saúde pública, de nível mundial, isso, devido as altas taxas de incidência, morbidade e mortalidade (YEUNG JH e GRAHAM CA, 2010).

De acordo com Silva VB (2017), no Brasil, em 2014 foram registradas 124.000 hospitalizações de crianças vítimas de acidentes que resultaram em traumas e fraturas ortopédicas, destas, 4.580 evoluíram à óbito, levando gastos de cerca de 83 milhões ao SUS.

Os traumas ortopédicos trazem interferências diretas na vida do sujeito ao qual este é submetido. Socialmente, o trauma e suas consequências físicas diretas ou até mesmo a amputação, impossibilita a pessoa de realizar atividades que antes eram normais para tal, levando a um estado de devaneio e nostalgia, que pode acarretar sequelas emocionais na vida do indivíduo, principalmente do indivíduo infantil, o qual ficará impossibilitado de exercer atividades sociais, como brincar ou praticar esportes, levando-os assim a uma constante irritação. (CASTRO RRM, et al., 2013; SOUZA DFM, et al., 2010)

Segundo Silva VB (2017), dentre os fatores que interferem na ocorrência do trauma, a idade é um dos mais importantes, levando em consideração que determinadas idades estão mais propensas a determinados traumas ortopédicos. Dentre tais, está a faixa etária pediátrica, a qual, a autora afirma ainda, estar mais propensas a eventos traumáticos de maior gravidade, levando em consideração sua maior fragilidade e menor tamanho. 
Acerca do público infanto-juvenil, o traumatismo é tido como a principal causa de morte de jovens, no mundo, ainda, quando não é fatal, o trauma pode afetar o desenvolvimento biopsicossocial da criança (WHO, 2018; ROSA JO, et al., 2018). De acordo com Ribeiro PGA (2015), as lesões traumáticas são comuns em infantes, sendo, possivelmente, provenientes das atividades físicas deste público. Estudos mostram que $20,25 \%$ das crianças sofrem de lesões, a cada ano (KELISHADI R, et al., 2014).

Por conseguinte, a prevalência de crianças do sexo masculino acometidas por traumas ortopédicos, observado neste estudo, está em consonância com os resultados relatados em outras pesquisas com temas semelhantes. No presente estudo, foi evidenciado que de um universo de 673 indivíduos, 375 (58,32\%), a maioria, eram meninos. No estudo de Rodrigues JB (2018) o sexo masculino também foi o mais predominante, com uma representação de $61,54 \%$ e no de Sousa GS, et al. (2019) a expressão foi de $69 \%$. No estudo de Sousa LRB, et al. (2017), na faixa etária de 0 a 11 anos, o grupo masculino fora responsável pelo maior índice, sendo ele $18,20 \%$ em comparação ao feminino, que na mesma idade foi de $9,70 \%$. Ainda, Silva VB (2017), em seu estudo sobre traumas ortopédicos infantis, demonstrou que $59,6 \%$ dos pacientes admitidos, eram do sexo masculino.

A importância dessa observação em maior prevalência de traumas em infantes do sexo masculino pode ser explicada de forma social. Culturalmente, as atividades lúdicas desempenhadas por crianças do sexo masculino possuem maior intensidade, o que aumenta o risco para acidentes com traumas ortopédicos. Também, estudos apontam o fato de que crianças do sexo masculino são mais ativas, o que os leva a se envolverem em aventuras mais perigosas. Também, a prática de esportes de maior impacto físico, é um dos fatores que os interpõe como mais predispostos a fraturas ortopédicas. (SANTILI C, et al., 2010; HEDSTROM EM, et al., 2010; SOUZA DFM, et al., 2010).

Quando analisamos as idades dos participantes desse estudo, foi observado que a faixa etária predominante foi a de crianças entre 5 a 12 anos (79,77\%). O estudo que aqui apresentamos, entra em consonância com a literatura, onde na pesquisa de Almeida M (2019), a idade mais acometida fora a de 10 - 14 anos, sendo 65,7\% da amostra do estudo. Franciozi CES, et al. (2008), demonstrou em seu estudo que, dentre a faixa etária infantil, a média de casos de fraturas ortopédicas, fica na idade de 10 anos. Ainda, Baron E et al. (2013), aponta que das amostras de idade de seu estudo sobre o tema aqui tratado, as crianças de 0 a 11 anos representaram a faixa etária mais atingida, sendo $47 \%$ do total.

Por conseguinte, devemos entender que a maior incidência nestas idades pode estar relacionada à atividade escolar, a acidentes de trânsitos com quedas (de bicicleta, por exemplo) e a prática esportiva (futebol, vôlei, basquete, entre outros) (SOUSA GS, et al., 2019; GUARNIERO R, et al., 2011; SANTILI C, et al., 2010).

Ainda, como já foi relatado em outros estudos sobre traumas ortopédicos em crianças e adolescentes, é perceptível que na faixa etária escolar os infantes começam a experimentar a independência e interação com o meio social, logo, tendem a se expor mais à possíveis traumas ortopédicos (PEREIRA IF, et al., 2013; SANTILI C, et al., 2010).

Ademais, fazendo uma reflexão acerca da procedência desses pacientes, percebemos que a maioria, 249 (38,73\%), é procedente do município de Tucuruí, tal fato, explica-se devido o hospital estar implantando no referido município. Para mais, o hospital é uma instituição de saúde pública estadual, que oferece serviços de alta e média complexidade, contando, atualmente, com 190 leitos e sendo a única instituição de referência em Urgência e Emergência na região do Lago de Tucuruí, oferecendo, portanto, seus serviços para mais de 400 mil habitantes (ROCHA EEM, et al., 2019). Nesse contexto, as demais procedências observadas no estudo são de municípios que possuem o hospital como referência no atendimento para trauma ortopédico.

No que tange a parte anatômica afetada pela lesão traumática, o estudo de Rosa JO, et al. (2018), afirma que os principais membros afetados foram os membros superiores, totalizando $60,99 \%$ dos casos, 0 que condiz com os resultados encontrados na presente pesquisa, na qual temos o rádio, antebraço, úmero e mãos, em evidências às outras partes afetadas, totalizando $72,26 \%$ do total de ocorrências cirúrgicas 
relacionadas a eventos traumáticos em pacientes pediátricos. O estudo de Franciozi CES, et al. (2008), também aponta os membros superiores como os mais atingidos, sendo o primeiro o Úmero $(20,9 \%)$ e 0 antebraço $(7,2 \%)$.

No estudo de Ribeiro PGA (2015), o qual se refere às fraturas ortopédicas pediátricas, é demonstrado que 33-37\% das lesões são localizadas no antebraço. Rodrigues JB (2018), conceitua fratura de antebraço como lesões que envolvem o rádio e a ulna, podendo ser somente um ou ambos, distal ou proximal, sendo decorrentes, geralmente, de quedas com a mão estendida. Ademais, a autora também afirma que as fraturas no antebraço têm maior predisposição a acontecerem, ao correlaciona-las a fatores como genética óssea, peso ao nascer e má nutrição.

Quanto aos acometimentos de membros inferiores do presente estudo, estes representaram $22,53 \%$ do total de procedimentos cirúrgicos realizados em pacientes pediátricos, a tíbia demonstrou-se como a mais propensa a sofrer lesões traumáticas, tendo $7,9 \%$ do total de ocorrências. Tais dados entram em consenso com o estudo de Santili C, et al. (2010), no qual, este afirma que das fraturas de membros inferiores, em crianças e adolescentes, as fraturas na tíbia são as mais comuns, representando de $10 \%$ a $15 \%$ de todas as fraturas na população pediátrica. $O$ autor, antes referido, frisa que as fraturas nessa região, são mais comuns em crianças que estão aprendendo a andar.

Seguida a tíbia, outra fratura recorrente na faixa-etária aqui tratada, é a fratura femoral, a qual representou $5,43 \%$ do total de procedimentos cirúrgicos. Hoffmann CR, et al. (2012), afirma ser uma das cirurgias de maior risco quanto à infecção, de maneira que a Sociedade de Ortopedia Pediátrica dos Estados Unidos, não recomendam o tratamento cirúrgico para pacientes menores de 06 anos, dando preferência ao método conservador de cuidado. $O$ autor, em sua pesquisa, demonstrou que as fraturas femorais que precisaram de procedimento cirúrgico, representaram cerca de $1,7 \%$ de todas as fraturas da população pediátrica.

Tratando-se de procedimentos cirúrgicos ortopédicos, no geral, a incidência de Infecções de Sítio Cirúrgico (ISC), se fazem de grande influência no processo de escolha do tratamento. Na população adulta, tal intercorrência tem como taxa de ocorrência, cerca de $11 \%$, e no que se refere à população pediátrica, essa taxa se dá $2,5 \%$ a $20 \%$ do total. Variáveis como idade, sexo, área afetada e tempo internação, têm influência direta na identificação casuística das ISC, dá-se, por isso, a importância do estudo epidemiológico dos procedimentos cirúrgicos ortopédicos pediátricos (CHAGAS MQL, et al., 2017; PERVIZI J, et al., 2013).

A revisão de literatura de escopo do trabalho, com o tema da epidemiologia de traumas ortopédicos, nos últimos 10 anos, no Brasil, se demonstrou escassa. De tal forma, a maioria dos perfis epidemiológicos formados, eram constituídos por pacientes de idades adultas. A formação de perfis epidemiológicos de pacientes pediátricos, como realizado no presente estudo, se faz de cerne importância para que profissionais utilizem destes, para a realização e direcionamento de público, no que tange às práticas de educação em saúde para com os pacientes e seus pais ou responsáveis. A determinação casuística, bem como a exposição dos indicadores, faz com que medidas preventivas se façam mais eficazes, cabendo aos profissionais de saúde a implementação de um sistema eficiente de educação continuada, relativo à segurança dos infantes, quanto prevenção de outros ou possíveis acidentes de cunho traumático, que possam vir a ser evitados (FRANCIOZI CES, et al., 2008; JUNIOR WL, et al., 2005; ARAÚJO NR, 2014).

\section{CONCLUSÃO}

Os resultados deste estudo apontaram para um número considerável de cirurgias ortopédicas pediátricas dentro de um período de 2 anos. Os principais acometidos foram meninos e, de um modo geral, crianças em idade escolar. Nesse contexto, devemos entender que tais achados são importantes, tendo em vista que o trauma ortopédico é considerado um problema de saúde pública e que, no público infantil, pode ocasionar mortes ou sequelas irreversíveis, de grau físico, social ou psicológico. Por conseguinte, qualificar e quantificar esses traumas cirúrgicos ortopédicos pediátricos, além de descrever o perfil dos pacientes atendidos pelo hospital, evidencia-se como uma estimulação para a realização de futuros estudos semelhantes, haja vista que é um tema pouco abordado pela literatura, no geral. 


\section{REFERÊNCIAS}

1. ALMEIDA M. Caracterização epidemiológica das admissões por trauma músculo-esquelético num serviço de urgência pediátrica de um hospital central. Revista Portuguesa de Ortopedia e Traumatologia, 2019; 27(1): 31-39.

2. ARAÚJO NR. Fatores Associados Ao Trauma Em Crianças E Adolescentes: Uma Revisão Narrativa, SC. Monografia - Especialização em Linhas de Cuidado em Enfermagem - Opção Urgência e Emergência. Universidade Federal de Santa Catarina, Florianópolis, 2014; 19 p.

3. BARON E, et al. Pediatric orthopedic injuries following an earthquake: experience in an acute-phase field hospital. Journal of trauma and acute care surgery, 2013; 74(2): 617-621.

4. CASTRO RRM, et al. Perfil dos pacientes da enfermaria de ortopedia de um hospital público de Salvador - Bahia. Acta Ortopédica Brasileira, 2013; 21(4): 191-194.

5. CHAGAS MQL, et al. Análise Das Infecções De Sítio Cirúrgico Em Pacientes Pediátricos Após Cirurgia Ortopédica: Um Estudo Caso-Controle. Revista Paulista de Pediatria, 2017; 35(1): 18-14.

6. CHAMLIAN TR, et al. Análise funcional e prognóstico de marcha no paciente amputado de extremidade inferior. Acta fisiátrica, 2013; 20(4): 200-206.

7. FRANCIOZI CES, et al. Trauma na infância e adolescência: epidemiologia, tratamento e aspectos econômicos em um hospital público. Acta Ortopédica Brasileira, 2008; 16(5): 261-265.

8. GUARNIERO R, et al. Estudo observacional comparativo de fraturas em crianças e adolescentes. Revista Brasileira de Ortopedia, 2011; 46: 32-37.

9. HEDSTROM EM, et al. Epidemiology of fractures in chidren and adolescents. Acta orthopaedica, 2010; 81(1): 148153.

10. HOFFMANN CR, et al. Estudo epidemiológico das fraturas femorais diafisárias pediátricas. Revista Brasileira de Ortopedia, 2012; 47(2): 186-190.

11. KELISHADI R, et al. Frequency, Causes and Places of Unintentional Injuries in a Nationally Representative Sample of Iranian Children and Adolescents: The CASPIAN-IV Study. International Journal of Preventive Medicine, 2014; 5(10): 1224-1230.

12. PARVIZI J, et al. Proceedings of the international consensus on periprosthetic joint infection. The bone \& joint jornal, 2013; 95(11): 1450-1452.

13. PEREIRA IF, et al. Perfil das internações de crianças e adolescentes com fraturas do crânio e ossos da face na região nordeste do Brasil. Rev bras ciênc saúde, 2013; 17(3): 275-80.

14. RIBEIRO PGA. O Tratamento Conservador das Fraturas na Criança. Dissertação (Mestrado Integrado em Medicina) - Faculdade de Medicina. Universidade do Porto, Porto, 2015. 43 p.

15. ROCHA EEM, et al. Diagnóstico do gerenciamento de resíduos de serviços de saúde: Hospital Regional de Tucuruí/PA. Anais do 30ํㅡㄹ Congresso Brasileiro de Engenharia Sanitária e Ambiental, 2019.

16. RODRIGUES JB. Pós-operatório pediátrico de fratura de antebraço: perfil e propostas de orientações. Dissertação (Mestrado em Enfermagem) - Programa de Pós-Graduação Strictu Sensu em Enfermagem. Faculdade de Medicina de São José do Rio Preto, São José do Rio Preto, 2018; 72p.

17. ROSA JO, et al. Epidemiologia do trauma ortopédico pediátrico em um hospital público. Revista Científica Multidisciplinar Núcleo do Conhecimento, 2018; 12(1): 166-173.

18. SANTILI C, et al. Fraturas da diáfise da tíbia em crianças. Acta ortop. Bras, 2010; 18(1); 45-48.

19. SANTOS LFS, et al. Estudo epidemiológico do trauma ortopédico em um serviço público de emergência. Cad. Saúde Colet., 2016; 24 (4): 397-403.

20. SILBERMAN FS, VARAONA O. Ortopedia y traumatologia. 4ª ed. Buenos Aires: Médica Panamericana, 2018; $624 p$.

21. SILVA LAP, et al. Análise retrospectiva da prevalência e do perfil epidemiológico dos pacientes vítimas de trauma em um hospital secundário. Revista de Medicina, 2017; 96(4): 246-54.

22. SILVA VB. Trauma pediátrico grave: análise da prevalência em hospital terciário do Distrito Federal, período de 2013 a 2015. Dissertação (mestrado)—Universidade de Brasília, Faculdade de Ciências da Saúde, Departamento de Enfermagem, Programa de Pós-Graduação em Enfermagem, 2017. 94P.

23. SOUZA DFM, et al. Epidemiologia das fraturas de face em crianças num pronto-socorro de uma metrópole tropical. Acta Ortopédica Brasileira, 2016; 18(6): 335-338.

24. SOUSA GS, et al. Epidemiologia dos acidentes com fraturas na infância: o retrato de um município da Amazônia brasileira. Revista Eletrônica Gestão \& Saúde, 2019; 68-80.

25. SOUSA LRB, et al. Notificação do acidente traumático em um hospital público da Amazônia brasileira. Revista Brasileira em Promoção da Saúde, 2017; 30(1): 64-71.

26. WORLD HEALTH ORGANIZATION (WHO). 2018. Global Reference List of 100 Core Health Indicators (plus healthrelated SDGs), 2018. Disponível em: https://www.who.int/healthinfo/indicators/2018/en/. Acesso em: 20/09/2020.

27. YEUNG JH, GRAHAM CA. Guidelines for trauma quality improvement programmes. European Jorunal of Emergence Medicine, 2010; 17(2): 101. 